\title{
Properties of extragalactic dust inferred from linear polarimetry of Type la Supernovae ${ }^{\star}$
}

\author{
F. Patat ${ }^{1}$, S. Taubenberger ${ }^{2}$, N. L. J. Cox ${ }^{3}$, D. Baade ${ }^{1}$, A. Clocchiatti ${ }^{4}$, P. Höflich ${ }^{5}$, J. R. Maund ${ }^{6}$, E. Reilly ${ }^{6}$, \\ J. Spyromilio ${ }^{1}$, L. Wang ${ }^{7}$, J. C. Wheeler ${ }^{8}$, and P. Zelaya ${ }^{4}$ \\ ${ }^{1}$ European Organisation for Astronomical Research in the Southern Hemisphere (ESO), Karl-Schwarzschild-Str. 2 , \\ 85748 Garching b. München, Germany \\ e-mail: fpatat@eso.org \\ 2 Max-Planck Institute for Astrophysics (MPA), Karl-Schwarzschild-Str. 1, 85748 Garching b. München, Germany \\ 3 Instituut voor Sterrenkunde, KU Leuven, Celestijnenlaan 200D, bus 2401, 3001 Leuven, Belgium \\ ${ }^{4}$ Departamento de Astronomia y Astrofisica, PUC, Casilla 306, 22 Santiago, Chile \\ 5 Department of Physics, Florida State University, Tallahassee, FL 32306-4350, USA \\ ${ }^{6}$ Queen's University Belfast, Belfast, BT7 1NN, UK \\ 7 Department of Physics, Texas A\&M University, College Station, TX 77843, USA \\ ${ }^{8}$ Department of Astronomy and McDonald Observatory, The University of Texas at Austin, Austin, TX 78712, USA
}

Received 1 July 2014 / Accepted 28 January 2015

\section{ABSTRACT}

\begin{abstract}
Aims. The aim of this paper is twofold: 1) to investigate the properties of extragalactic dust and compare them to what is seen in the Galaxy; 2) to address in an independent way the problem of the anomalous extinction curves reported for reddened Type Ia Supernovae (SN) in connection to the environments in which they explode.

Methods. The properties of the dust are derived from the wavelength dependence of the continuum polarization observed in four reddened Type Ia SN: 1986G, 2006X, 2008fp, and 2014J. The method is based on the observed fact that Type Ia SN have a negligible intrinsic continuum polarization. This and their large luminosity makes them ideal tools to probe the dust properties in extragalactic environments.

Results. All four objects are characterized by exceptionally low total-to-selective absorption ratios $\left(R_{V}\right)$ and display an anomalous interstellar polarization law, characterized by very blue polarization peaks. In all cases the polarization position angle is well aligned with the local spiral structure. While SN 1986G is compatible with the most extreme cases of interstellar polarization known in the Galaxy, SN 2006X, 2008fp, and 2014J show unprecedented behaviours. The observed deviations do not appear to be connected to selection effects related to the relatively large amounts of reddening characterizing the objects in the sample.

Conclusions. The dust responsible for the polarization of these four SN is most likely of interstellar nature. The polarization properties can be interpreted in terms of a significantly enhanced abundance of small grains. The anomalous behaviour is apparently associated with the properties of the galactic environment in which the SN explode, rather than with the progenitor system from which they originate. For the extreme case of SN 2014J, we cannot exclude the contribution of light scattered by local material; however, the observed polarization properties require an ad hoc geometrical dust distribution.
\end{abstract}

Key words. supernovae: general - supernovae: individual: 1986G - supernovae: individual: 2006X - supernovae: individual: 2008fp supernovae: individual: $2014 \mathrm{~J}$ - dust, extinction

\section{Introduction}

Studying cosmic dust has important consequences on our understanding of a number of astrophysical processes, ranging from galaxy evolution to stellar and planetary formation. The characterization of dust in the diffuse interstellar medium relies heavily on the observed wavelength dependencies of extinction and polarization (see Voshchinnikov 2012, for a comprehensive review). While this study can be undertaken in our Galaxy using pair-matching of stars of the same spectral and luminosity classes (see Whittet 2003, for a review), its application to the extragalactic case is only possible for very few nearby systems, like

$\star$ Based on observations made with ESO Telescopes at the Paranal Observatory under Program IDs 076.D-0177 and 076.D-0178. This study is also based on observations collected at the German-Spanish Astronomical Center, Calar Alto (Spain) and on observations made with the Mercator Telescope, Roque de los Muchachos, La Palma (Spain) equipped with the HERMES spectrograph. the Magellanic clouds. At larger distances, other methods are used: foreground extinction of distant quasars, differential extinction of multiply lensed quasars, gamma-ray-burst/supernova host extinction, and extinction in star-burst galaxies.

In this article, we focus on the use of Type Ia Supernovae (SN). These bright $\left(M_{V} \sim-19\right)$ objects were used several times as line-of-sight probes to infer the properties of the intervening material. The technique applied so far can be considered as an extension of the pair-matching method, in which the observed spectrophotometric properties are compared to a set of un-reddened templates. This has led to the striking result that the total-to-selective absorption ratio $\left(R_{V}\right)$ is systematically and significantly lower than what is typical in the Galaxy $\left(R_{V} \sim 3\right.$; Fitzpatrick \& Massa 2007). From the analysis of 80 Type Ia SN with $E_{B-V} \leq 0.7$, Nobili \& Goobar (2008) derived an average value $R_{V}=1.75 \pm 0.27$. Although this result was revised towards more "normal" values $\left(R_{V}=2.8 \pm 0.3\right.$, Chotard et al. $2011)$, objects with relatively large reddening $\left(E_{B-V}>0.4\right)$ show 
Table 1. Basic properties of the SN sample.

\begin{tabular}{llllllllllll}
\hline \hline SN & Host & $\begin{array}{l}\text { Host } \\
\text { type }\end{array}$ & $\begin{array}{l}\text { Host } \\
\text { notes }\end{array}$ & $R_{V}$ & $A_{V}$ & $E_{B-V}$ & Ref. $\begin{array}{c}P_{04} \\
(\%)\end{array}$ & $\begin{array}{c}\lambda_{\max } \\
(\mu \mathrm{m})\end{array}$ & $\begin{array}{c}P_{04} / E_{B-V} \\
\left(\% \mathrm{mag}^{-1}\right)\end{array}$ & $\begin{array}{c}\theta \\
(\mathrm{deg})\end{array}$ \\
\hline 1986G & NGC 5128 & S0, pec & starburst & $2.57_{-0.21}^{+0.23}$ & $2.03_{-0.13}^{+0.09}$ & $0.79_{-0.08}^{+0.08}$ & 1 & $5.1 \pm 0.1$ & 0.43 & $6.5 \pm 0.7$ & $117.9 \pm 0.8$ \\
2006X & NGC 4321 & SAB(s)bc & & $1.31_{-0.10}^{+0.08}$ & $1.88_{-0.13}^{+0.09}$ & $1.44_{-0.15}^{+0.11}$ & 1 & $7.8 \pm 0.2$ & $<0.4$ & $5.4 \pm 0.5$ & $139.5 \pm 0.1$ \\
2008fp & ESO 428-G14 & SAB(r)0 & active & $1.20_{-0.14}^{+0.26}$ & $0.71_{-0.08}^{+0.10}$ & $0.59_{-0.10}^{+0.15}$ & 1 & $2.6 \pm 0.1$ & $<0.4$ & $4.4 \pm 1.0$ & $148.4 \pm 0.2$ \\
2014J & NGC 3034 & I0 & starburst & $1.40 \pm 0.10$ & $1.85 \pm 0.11$ & $1.37 \pm 0.03$ & 2 & $6.6 \pm 0.1$ & $<0.4$ & $4.8 \pm 0.1$ & $42.2 \pm 0.3$ \\
\hline
\end{tabular}

References. (1) Phillips et al. (2013); (2) Amanullah et al. (2014).

unusually low $R_{V}$ (Phillips et al. 2013). This has interesting implications both for our understanding of dust and the explosion environments of Type Ia SN.

Here we discuss an independent approach, which relies on the fact that SN Ia have a very low intrinsic continuum polarization $(<0.3 \%$, Wang \& Wheeler 2008). This level of continuum polarization is negligible when the reddening is significant $\left(E_{B-V}>0.1-0.2\right)$ and the interstellar polarization prevails. Their luminosity, insignificant intrinsic continuum polarization, and the supposed absence of significant amounts of circumstellar material (at variance with core-collapse events) with possibly peculiar characteristics, make Type Ia SN suitable background illuminators to probe the dust properties in extragalactic environments.

The paper is structured as follows. In Sect. 2 we present the sample. Sect. 3 discusses the continuum polarization dependence and compares it to a sample of Galactic stars. The significance of the observed deviations from the Galactic behaviour and their physical implications are analysed in Sect. 4. Finally, our conclusions are summarized in Sect. 5.

\section{Data}

The number of reddened Type Ia $\mathrm{SN}\left(E_{B-V}>0.5\right)$ with polarimetric data of sufficient quality (in terms of accuracy and wavelength coverage) is very small. To the best of our knowledge this includes only SN 1986G (NGC 5128; Cen A), 2006X (NGC 4321; M100), 2008fp (ESO 428-G14), and the recent 2014J (NGC 3034; M 82). Other Type Ia SN have spectropolarimetric data: 1997dt, 2002bf, 2003du, 2004dt (Leonard et al. 2005); 2001el (Wang et al. 2003); 2007sr (Zelaya et al. 2013); 2005ke (Patat et al. 2012); 2011fe (Smith et al. 2012); 2012fr (Maund et al. 2013); however, the low reddening associated with these events makes the study of the interstellar polarization (ISP) wavelength dependence very uncertain, if not impossible (see also Zelaya et al., in prep.).

The four selected objects show a pronounced continuum polarization at position angles remarkably well aligned with the local spiral arms of their hosts, as expected for dust grain alignment along the galactic magnetic field (Scarrot et al. 1987). In all cases, the wavelength dependence of the polarization position angle is very mild or null (see Fig. 1), similar to what is seen in Galactic stars (Dolan \& Tapia 1986). All four SN Ia have high-quality, high-resolution spectroscopy data, with a number of separate interstellar absorption components indicating significant amounts of gas (atomic and molecular) at different velocities along the line-of-sight (D'Odorico et al. 1989; Cox \& Patat 2008, 2014; Cox et al. 2014; Goobar et al. 2014; Welty et al. 2014).

The relevant properties of the sample discussed in this paper are summarized in Table 1. In addition to the reddening

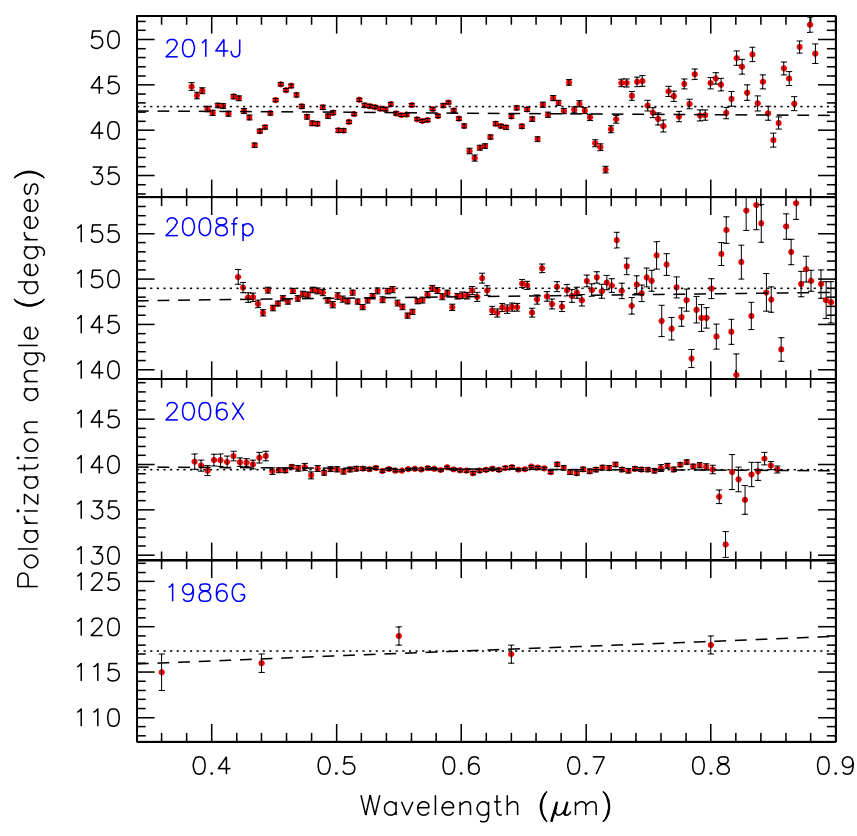

Fig. 1. Polarization angles for the $\mathrm{SNe}$ in the sample. The dashed lines trace the weighted linear best fit $(\lambda \leq 0.8 \mu \mathrm{m})$, while the dotted lines indicate the average value computed using the full wavelength range.

characteristics, the table reports the basic polarization parameters, which will be introduced and discussed in Sect. 3 .

\subsection{SN 1986G, 2006X, and 2008fp}

SN 1986G is the first Type Ia SN that was used for studying the polarization properties of the intervening dust. For this object, UBVRIJH broad-band polarimetry was obtained by Hough et al. (1987). SN 2006X and 2008fp were observed in the framework of our spectropolarimetric programme with the FOcal Reducer/low-dispersion Spectrograph (FORS1), mounted at the Cassegrain focus of the ESO-Kueyen $8.2 \mathrm{~m}$ telescope (Appenzeller et al. 1998). The data and their reduction are discussed in Patat et al. (2009) and Cox \& Patat (2014).

\subsection{SN 2014J}

Spectropolarimetry of SN 2014J is reported here for the first time. A preliminary analysis was published by Patat et al. (2014). A full analysis of the spectropolarimetric data will be presented in a separate paper, while here we focus purely on the continuum polarization.

We obtained linear spectropolarimetry of SN 2014J on three epochs (Jan. 28, Feb. 03, and Mar. 08, 2014), using the Calar Alto Faint Object Spectrograph (CAFOS), mounted at the $2.2 \mathrm{~m}$ 
telescope in Calar Alto, Spain (Meisenheimer 1998; Patat \& Taubenberger 2011, hereafter PT11). We obtained all spectra with the low-resolution B200 grism coupled with a 1.5 arcsec slit, giving a spectral range 3300-8900 $\AA$, a dispersion of $\sim 4.7 \AA \mathrm{px}^{-1}$, and a full width half maximum (FWHM) resolution of $21.0 \AA$. For each retarder angle, we obtained two exposures; integration times ranged from 10 to 25 min per exposure. Because of the low signal-to-noise ratio and the known instrumental limitations (PT11), the CAFOS spectropolarimetric data below $3800 \AA$ A cannot be used.

The Stokes parameters were derived with the same procedure as used for SN 2006X and 2008fp. We peformed error estimates following the prescriptions described by Patat \& Romaniello (2006), while the HWP zero-point angle chromatism was corrected using tabulated data (PT11). To check and correct for instrumental polarization, we observed the unpolarized standard HD 90508 (Serkowski 1974) at each epoch. We detected spurious polarization of $0.3 \%$. Although the polarization level of HD 90508 is constant $(\sim 0.25 \%$; see also PT11), different corrections were applied to the three data sets, as the polarization angle changed between the epochs. For this reason, the correction is accurate to a few $0.1 \%$. Given the high level of polarization displayed by SN 2014J (see below), this does not affect any of the conclusions that we present. The overall performance of the system and the quality of the chromatic and instrumental polarization corrections were checked using observations of the polarized standard star HD 43384 (Mathewson \& Ford 1970). The maximum polarization is $P_{\max }=3.10 \pm 0.05 \%$ at $\lambda_{\max }=0.539 \mu \mathrm{m}$ and the average position angle $\theta=170.3 \pm$ $0.2 \mathrm{deg}$. These values are fully consistent with those reported by Serkowski et al. (1975): $P_{\max }=3.0 \%, \lambda_{\max }=0.53 \mu \mathrm{m}$, $\theta=170 \mathrm{deg}$. The marked wavelength dependence of the position angle $\left(\mathrm{d} \theta / \mathrm{d} \lambda=7.4 \pm 0.6 \mathrm{deg} \mu \mathrm{m}^{-1}\right)$ is consistent with the findings of Doland \& Tapia (1986).

For our purposes, we use the data of the second epoch that, being obtained closest to maximum light, have the best signalto-noise ratio. The first and the last epoch show very similar behaviour, with the changes related to the evolution of the intrinsic SN properties, most prominently in the Si II $6355 \AA$ and the Ca II near-IR triplet. The continuum polarization level is constant within the measurement errors.

The signal is dominated by a continuum polarization that grows steadily and rapidly from the red to the blue, reaching about $6.6 \%$ at $0.4 \mu \mathrm{m}$. The average polarization position angle for $\mathrm{SN} 2014 \mathrm{~J}$ is $42.2 \pm 0.3 \mathrm{deg}$, which is well aligned with the local spiral structure and in good agreement with the value reported by Greaves et al. (2000) for the dust lane (40 deg). In particular, it is fully consistent with the position angle distribution along the dust lane of M 82 (Jones 2000; see his Figs. 4 and 5) and identified as being generated by transmission (as opposed to scattering).

\section{ISP wavelength dependence}

In Fig. 2 we present the wavelength dependence displayed by the four SN. The deviation from the typical behaviour seen in the Milky Way (MW) is illustrated by the comparison with the Galactic star HD 43384 (solid histogram). While SN 1986G shows a polarization maximum at $\lambda_{\max }=0.435 \mu \mathrm{m}, \mathrm{SN} 2006 \mathrm{X}$, 2008fp, and 2014J do not show evidence of a peak in the spectral range covered by the data. In the absence of a maximum, we quantify the polarization level with the value measured at $0.4 \mu \mathrm{m}$ $\left(P_{04}\right)$. This is then used to compute the polarization efficiency
$P_{04} / E_{B-V}$, which ranges from 4.4 to $6.5 \% \mathrm{mag}^{-1}$ (see Table 1). Since the polarization maximum is not observed, these are only lower limits for the true polarization efficiency. It is therefore not possible to firmly establish whether or not the SN conform to the Galactic limit $P_{\max } / E_{B-V}<9 \% \mathrm{mag}^{-1}$ (Serkowski et al. 1975). The weighted average $P_{04} / E_{B-V}$ is $4.9 \pm 0.4 \% \mathrm{mag}^{-1}$. Given the relatively large values of $E_{B-V}$, this estimate is robust in contrast to low-extinction cases (see the discussion in Leonard et al. 2002). For presentation, the polarization curves are normalized to $P_{04}$. The errorbars were kept at their original amplitudes to allow for a direct comparison of the different uncertainties characterizing the various data sets.

The exceptional behaviour displayed by Type Ia SN compared Galactic stars is made more evident by considering the wavelength dependence of the most extreme known cases. To the best of our knowledge, HD 193682 has the bluest ISP peak reported in the literature, with $\lambda_{\max } \leq 0.32 \mu \mathrm{m}$ (Anderson et al. 1996); however, a more recent study revised this finding, repositioning the maximum at $0.445 \pm 0.020 \mu \mathrm{m}$ (Weitenbeck 2008). Other interesting cases are those of Cyg OB2 No. 10 and 12, for which Whittet et al. (1992) report $\lambda_{\max }=0.33 \mu \mathrm{m}$ and $0.35 \mu \mathrm{m}$, respectively. The polarization wavelength dependence seen in these two objects resembles that of SN 1986G, possibly with a larger slope (see also the best fit presented by Martin et al. 1992 for Cyg OB2 No. 12: $\lambda_{\max }=0.35 \pm 0.02 \mu \mathrm{m}$, $K=0.61 \pm 0.04$ ). Another relevant case is that of HD 210121, for which independent estimates of polarization and total-toselective extinction are available. This star was reported to have $\lambda_{\max }=0.38 \pm 0.02 \mu \mathrm{m}$ and an exceptionally low $R_{V}=2.1 \pm 0.2$ (Larson et al. 1996). In general, the wavelength dependence is again similar to that of SN 1986G. In the optical and near-UV, the two data sets are consistent within the one-sigma errors (see Fig. 2, gray filled circles), while the near-IR data indicate a slightly flatter curve for HD 210121.

As already discussed in Patat et al. (2009), SN 2006X shows a steadily growing polarization to the blue, reaching about $8 \%$ at the blue edge of the spectral coverage. The curve flattening observed in the blue suggests that the maximum polarization occurs shortly before $0.38 \mu \mathrm{m}$. A non-linear least-squares fit using the Serkowski empirical expression $\left(P(\lambda) / P_{\max }=\right.$ $\left.\exp \left[-K \ln ^{2}\left(\lambda_{\max } / \lambda\right)\right]\right)$ gives $K=1.47 \pm 0.05$ and $\lambda_{\max }=0.365 \pm$ $0.02 \mu \mathrm{m}$. Although the derived value for $K$ is completely different from that predicted by the Whittet et al. (1992) relation $(0.62 \pm 0.07)$, the resulting curve gives an excellent match to the data (see Fig. 2).

The case of SN 2008fp is similar to that of SN 2006X (see also Cox \& Patat 2014); however, the nature of the data and the increased noise in the red (partially due to a marked fringing) do not allow a well-constrained fitting with a Serkowski law (the formal solution is rather degenerate, with very elongated contours in the $\chi^{2}$ space). We note that probably one third of the reddening suffered by this object arises in the Milky Way (see also Cox \& Patat 2014), so that the red portion of the curve may be affected by the Galactic component. The slope observed in the blue favours a value of $K$ smaller than that of 2006X, with a possible maximum below $0.4 \mu \mathrm{m}$. A formal least-squares fit using the Serkowski formulation yields $K=0.40 \pm 0.05$ and $\lambda_{\max }=0.148 \pm 0.03 \mu \mathrm{m}$.

Finally, SN 2014J appears to be the most extreme case, with a very steep polarization increase in the blue. With the lack of UV data, it is impossible to extrapolate the position of the polarization peak. The Serkowski law was shown to approximately hold in the UV for Galactic stars with $\lambda_{\max }$ in the optical and UV coverage (see Anderson et al. 1996; Martin et al. 1999). 


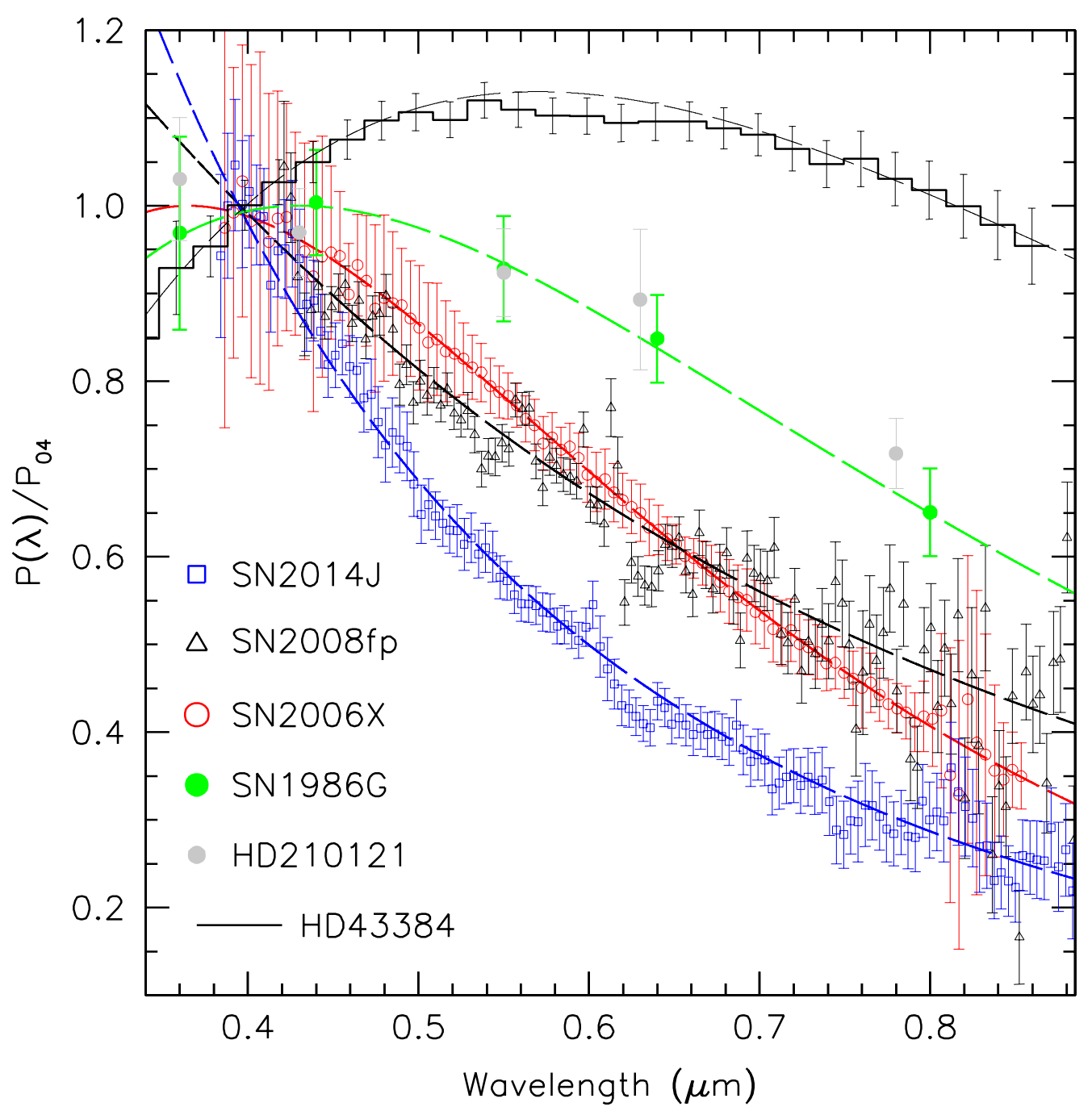

Fig. 2. Observed polarization wavelength dependence for SN 1986G (Hough et al. 1987), SN 2006X (Patat et al. 2009), SN 2008fp (Cox \& Patat 2014), and SN 2014J (this work). For comparison, the data of HD 43384 (this work) and HD 210121 (Larson et al. 1996) are also plotted. The dashed curves trace Serkowski law best fits. For presentation, the curves were normalized to $P_{04}$ (see text). The error bars were kept at their original values. HD 43384 is a polarized standard; HD 210121 is a Galactic star with very low $R_{V}$. For SNe 2006X, 2008fp and 2014J, $P(\lambda)$ was computed as the polarization component parallel to the polarization angle.

However, with only the possible exception of HD 193682, there are no published direct observations of Galactic objects with peaks in the UV. The best fit for SN 2014J has $K=0.40 \pm 0.07$ and $\lambda_{\max }=0.05 \pm 0.02 \mu \mathrm{m}$. For both SN 2008fp and 2014J, the derivation of the Serkowski parameters is based on the unjustified assumption that the expression can be extrapolated to extremely short wavelengths. The best-fit curves are plotted in Fig. 2 for pure illustrative purposes (see next section).

In summary, with the possible exception of SN 1986G, the wavelength dependence seen in our SN sample has never been observed in the Galaxy. A similar conclusion was reached by Kawabata et al. (2014), based on the published data for 1986G, 2006X and 2008fp and broad-band polarimetry for 2014J.

\section{Discussion}

\subsection{Statistical significance}

Although we have shown with some confidence that all four $\mathrm{SN}$ in our sample deviate from the average behaviour seen in the Galaxy, it is important to quantify the significance of this deviation. For this purpose, in Fig. 3 we present the Galactic samples by Whittet et al. (1992) and Voshchinnikov et al. (2013) on the $\lambda_{\max }-K$ plane. The second sample has some intersection with the first and no error bars are given. It is therefore included only for the sake of completeness and it is not used in the statistical analysis that follows.

As concluded by Whittet et al. (1992; see also Wilking et al. 1982), the Galactic data cluster around a linear relation $(K=$ $0.01 \pm 0.05+[1.66 \pm 0.09] \lambda_{\max }$ ). This empirical law (see Fig. 3, long-dashed line) was derived by Whittet et al. (1992) rejecting 27 objects from the original sample that includes 105 stars. For quantifying the bi-dimensional distribution, we have estimated the probability contours from the error-weighted covariance matrix computed on the full Whittet et al. sample. These are traced as light-colored ellipses at 1-sigma intervals. The misalignment between the best-fit Whittet et al. relation and the major axis of the ellipses is due to the inclusion of the 27 objects rejected by Whittet et al. (1992).

Out of the total sample, $1.9 \%$ of the Galactic objects fall outside of the 4-sigma level. Of the five stars with $\lambda_{\max }<$ $0.4 \mu \mathrm{m}$, four have $K$ values that are consistent with the derived relation. The other, Cyg OB $2 \mathrm{~A}$, is off by 4 sigma 
F. Patat et al.: Properties of extragalactic dust inferred from linear polarimetry

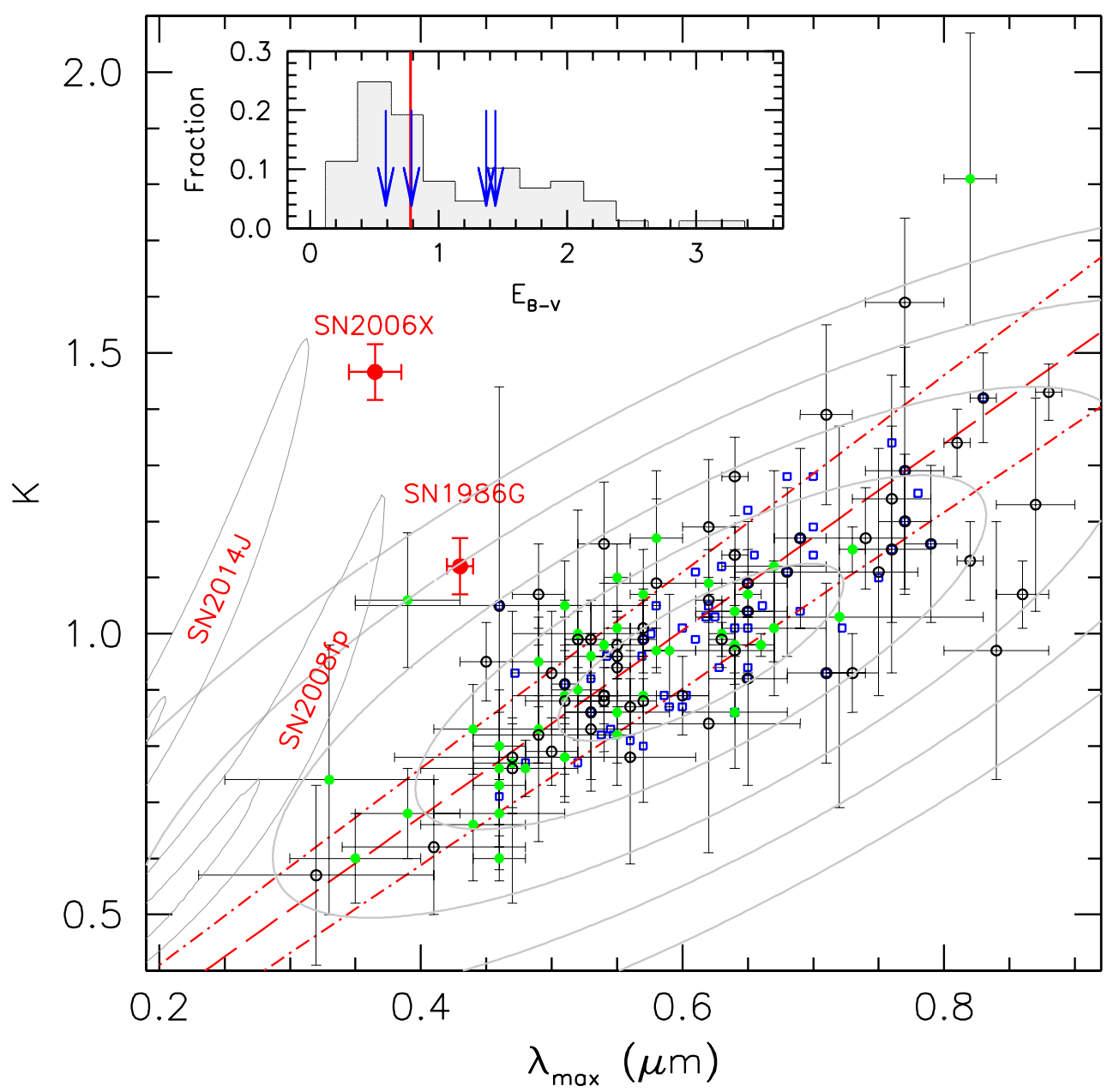

Fig. 3. Serkowski parameters $\lambda_{\max }$ and $K$ for Galactic stars. The data are from Whittet et al. (1992, circles) and Voshchinnikov et al. (2013, squares). Filled symbols indicate objects with $E_{B-V}>0.8$. The ellipses trace the 1- to 5-sigma confidence levels for the full Whittet et al. sample (for a bi-variate Gaussian distribution). For comparison, the parameters derived for SN 1986G and 2006X are plotted (large filled circles). The long-dashed line traces the Whittet et al. relation (the dashed-dotted lines indicate the \pm 1 -sigma levels). The contours in the lower left corner of the plot indicate the 10- and 20-sigma confidence levels for SN 2008fp and 2014J. The upper left insert plots the $E_{B-V}$ distribution of the Whittet et al. (1992) sample of 105 stars. The vertical line marks the median value of $E_{B-V}$, while the arrows indicate the values of the four SN (see Table 1).

$\left(\lambda_{\max }=0.39 \pm 0.04 \mu \mathrm{m}, K=1.06 \pm 0.12 ; E_{B-V}=2.59\right)$. Interestingly, for this star Whittet et al. (1992) report that it "has a polarization curve rising anomalously steeply to the blue, and lies furthest from the best straight line". Although the wavelength dependence is very similar to that shown by SN 1986G, for this star there are no $U$-band data and the B point is characterized by a relatively large uncertainty $(P=6.6 \pm 0.7 \%)$. At face value, there are no signs of a maximum in the optical domain for this star.

Cy OB 2 A represents slightly less than $1 \%$ of the Whittet et al. (1992) sample. This fraction decreases to $\sim 0.7 \%$ if one includes the Voshchinnikov et al. (2013) sample, giving a first quantitative estimate of the rarity of SN 1986G-like polarizations in our Galaxy. As first pointed out by Hough et al. (1987), this indicates that the dust along the line of sight to SN $1986 \mathrm{G}$ is significantly different from what has been studied and published so far for the MW. SN 2006X constitutes an even more deviant case (Patat et al. 2009). The polarization law for this SN appears to be at more than 9 sigma from the MW sample (see Fig. 3).

The cases of 2008fp (Cox \& Patat 2014) and 2014J are more difficult to place on the $\lambda_{\max }-K$ plane, because of the degeneracy of the Serkowski law fitting caused by very weakly constrained peak positions (see previous section). Nevertheless, one can get an indication by running a brute-force $\chi^{2}$ mapping as a function of $\lambda_{\max }$ and $K$. The resulting 10- to 20-sigma confidence levels are plotted in Fig. 3 (left lower corner). As anticipated, they are very elongated, hence implying a wide range of suitable parameter combinations. Although this is strictly valid only for a Serkowski law (and there is no guarantee that this holds when the maximum is in this extremely blue regime), the contour plots indicate that both objects deviate in a very significant way from the Galactic behaviour. Given the similarities between SN 2006X and 2008fp (see Fig. 2), we argue that SN 2008fp deviates from the Galactic sample at a similar statistical significance level.

This analysis confirms the findings presented in the previous section: all four $\mathrm{SN}$ in our sample display exceptional properties. If they were all drawn from the Galactic distribution, then having four outlier events at the observed significance levels would be very unlikely. At face value this leads to the conclusion that they belong to a different underlying population.

\subsection{Selection effects}

The current sample of $\mathrm{SN}$ is small and possible selection effects may be playing an important role. Because of the way 

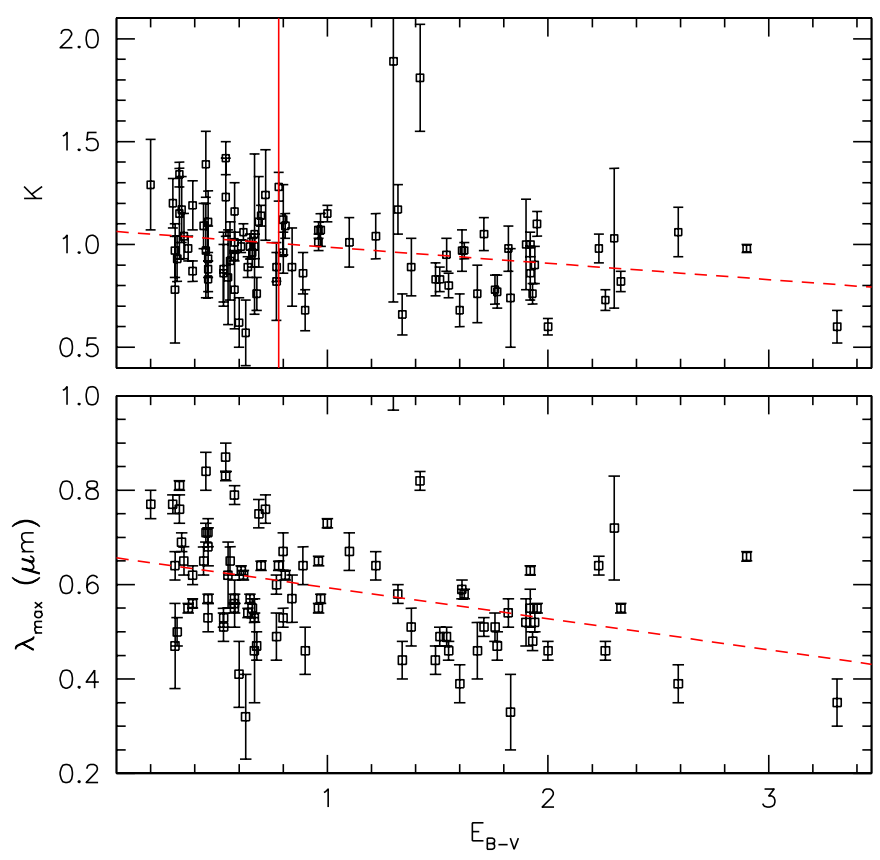

Fig. 4. Serkowski parameters $K$ and $\lambda_{\max }$ as a function of $E_{B-V}$ for the Whittet et al. (1992) sample. The dashed lines trace the best linear leastsquares fit to the data. The vertical solid line marks the median $E_{B-V}$.

the sample was constructed, the four $\mathrm{SN}$ discussed in this paper are all significantly reddened $\left(0.6 \lesssim E_{B-V} \lesssim 1.4\right.$; see Table 1$)$. One may therefore argue that this introduces systematic biases with respect to the Galactic sample, hence favouring special $\lambda_{\max }, K$ combinations. The $E_{B-V}$ distribution for the Whittet et al. sample is shown in the insert of Fig. 3 and has a median $E_{B-V} \simeq 0.8$. The stars with a reddening larger than the median tend to show smaller values of $\lambda_{\max }$ and $K$ (see the filled circles in Fig. 3). However, the linear correlation coefficients of $\lambda_{\max }$ and $K$ vs. $E_{B-V}$ are -0.34 and -0.24 , respectively, indicating a weak dependence of these parameters on the reddening (see Fig. 4). In addition, $\sim 30 \%$ of the Whittet et al. stars have $E_{B-V}>1.4$, i.e. larger than the highest value in the SN sample (see Table 1). These facts imply that, at least for the Galactic sample, selecting highly reddened objects does not lead to strong systematic effects on the final determination of the $K$ vs. $\lambda_{\max }$ relation, or on the overall distribution of the selected events on the $\lambda_{\text {max }}-K$ plane.

We note that the ISP wavelength dependence observed in several supernovae, not only of Type Ia, clearly deviates from the Galactic Serkowski law (Leonard \& Filippenko 2001; Maund et al. 2007, 2010; Patat et al. 2010). Notably, the Type II SN 1991gi shows a normal wavelength dependence $(K=1.15$ and $\lambda_{\max }=0.51 \mu \mathrm{m}$, Leonard et al. 2002). This SN suffered a minimal reddening $\left(E_{B-V}=0.21 \pm 0.09\right.$, with a robust upper limit of $\left.E_{B-V}<0.45\right)$. Although in the light of the above findings this may be more an exception than the rule, the low reddening experienced by this object may again indicate that the strong deviations occur in highly obscured events.

Another possible source of bias resides in the host characteristics. Both NGC 5128 (S0, peculiar) and NGC 3034 (I0, edge on) are starburst galaxies, a fact that may have some relevance for the dust properties (see for instance Hutton et al. 2014). While SN 2014J in NGC 3034 is the most discordant object, SN 1986 G in NGC 5128 is the least deviant. The host of
SN 2006X is a normal SAB(s)bc spiral, while 2008fp exploded in a peculiar $\mathrm{SAB}(\mathrm{r}) 0$ galaxy, with radio jets and signs of activity (Veron \& Veron 2006). Given the exiguous size of the sample, it is impossible to tell whether there is a systematic correlation between the host properties and the ISP behaviour.

Finally, one can question whether the observed behaviours are due to the peculiar nature of isolated clouds along the linesof-sight. In both SN 2008fp and 2006X (that incidentally show a similar ISP wavelength dependence), the bulk of reddening is produced within molecular clouds signaled by exceptionally strong CN features (Cox \& Patat 2008, 2014; see also the discussion in Phillips et al. 2013). Molecular gas was also detected towards SN $1986 \mathrm{G}$ in the form of weak $\mathrm{CH}$ and $\mathrm{CH}^{+}$absorptions, although no $\mathrm{CN}$ features were reported (D'Odorico et al. 1989). The situation is very similar for SN 2014J, in which CN is very weak (Welty et al., 2014; Sternberg et al., in prep.).

In the case of $2006 \mathrm{X}$ and $2008 \mathrm{fp}$, the bulk of reddening (and hence polarization) most probably originates within one single large thick cloud, which is not the case for SN 1986G and 2014J. For these two objects high-resolution spectroscopy reveals a number of $\mathrm{Na}$ I and $\mathrm{Ca}$ II features with comparable depth. This is illustrated for 2014J in Fig. 5, which presents the absorption profiles of $\mathrm{Na}$ and $\mathrm{K}$ lines (see also Welty et al. 2014). In addition to the Galactic component at zero velocity, the profile shows more than ten extragalactic absorptions. Even within the strongly saturated $\mathrm{Na}$ I D feature, the weaker K I line reveals more than five distinct components of comparable strength. This excludes that the bulk of reddening (and polarization) is generated within one single thick cloud with peculiar properties. The observed behaviour results from the combined presence of numerous clouds along the line-of-sight, spanning a wide range of velocities (and most probably positions) within the host. For this reason, we conclude that what is observed in SN 2014J reflects the average properties of the disk of M 82, which is seen almost edge-on. This conclusion probably also applies to SN 1986G, which displays a similarly large number of components (D'Odorico et al. 1989).

\subsection{Implications on dust properties and extinction law}

Before addressing the implications of the findings presented in the previous sections, it is important to note that deviations from the empirical Serkowski law can be obtained by combining the effects of two or more clouds with different properties (see Coyne \& Gehrels 1966; see also Patat et al. 2010; Appendix B). For instance, one can imagine a case in which the observed polarization results from the combination of two dust systems, individually described by different combinations of $P_{\max }, \lambda_{\max }, K$ and position angle $\theta$. Depending on the values of these parameters, the output polarization may show very deviant wavelength dependencies. Normally this is accompanied by a wavelength dependence of the position angle (like the one observed for HD 43384; see Sect. 2). Hough et al. (1987) reported a mild dependence $\left(\mathrm{d} \theta / \mathrm{d} \lambda=4.5 \pm 1.9 \mathrm{deg} \mu \mathrm{m}^{-1}\right)$ for 1986G, which may signal that the polarization position angles of the various clouds along the line of sight are different. For the other objects in the sample, $\mathrm{d} \theta / \mathrm{d} \lambda$ is consistent with zero (2006X: $-0.8 \pm 0.5 \operatorname{deg} \mu \mathrm{m}^{-1} ; 2008 \mathrm{fp}:+1.7 \pm 1.4 \mathrm{deg} \mu \mathrm{m}^{-1}$; 2014J: $-0.9 \pm 1.6 \mathrm{deg} \mu \mathrm{m}^{-1}$; see also Fig. 1). It is important to note that there is no way any combination of Serkowski laws with $\lambda_{\max }$ in the wavelength interval found in our Galaxy can produce polarization curves like those observed for SN 2006X, 2008fp, and 2014J. This implies that there must be different reasons for the observed behaviour. 


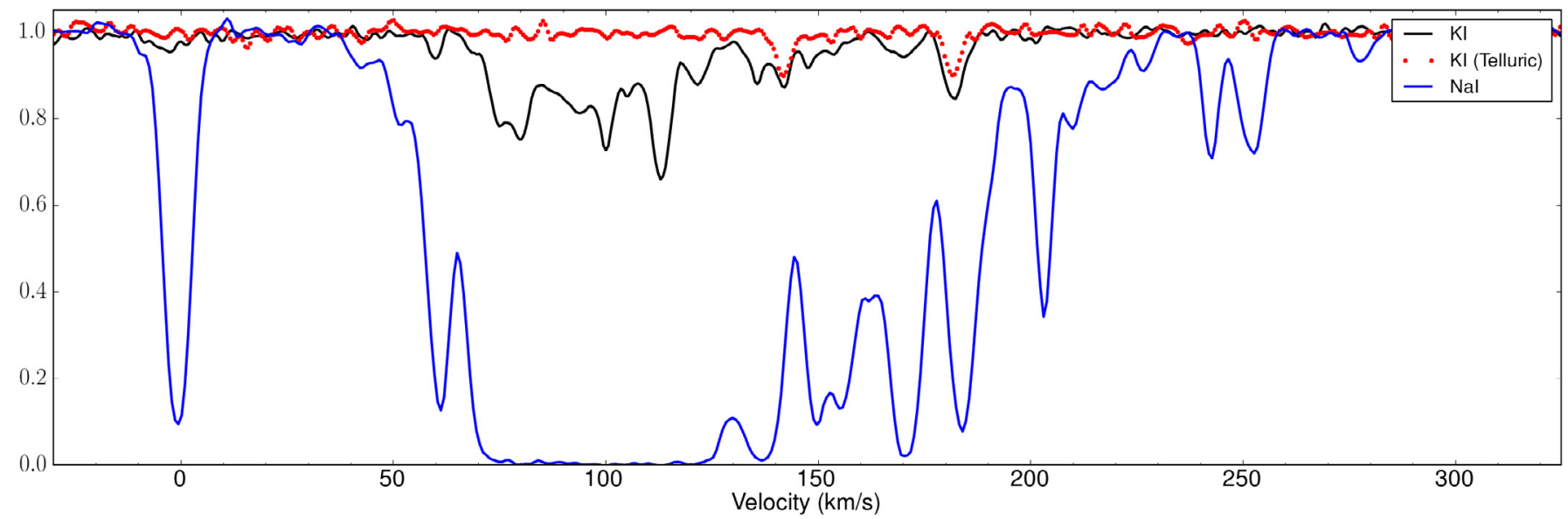

Fig. 5. Galactic and interstellar absorption components for Na ID (blue) and K I (black). The dotted curve traces a telluric absorption spectrum for the K I region. The data were obtained with the HERMES spectrograph (Sternberg et al., in prep.)

The observed linear dependence of $K$ on $\lambda_{\max }$ is qualitatively understood as a systematic increase in the number of small, aligned grains in regions displaying bluer polarization peaks (Martin et al. 1999; Whittet et al. 2001; Voshchinnikov 2012). This argument has been used, for instance, to infer that the size of dust grains along the line of sight to SN 1986G is $\sim 20 \%$ smaller than what is typical in the MW (Hough et al. 1987). This is more quantitatively confirmed by recent modelling. Siebenmorgen et al. (2014; their Fig. 5) show that substantial changes in the polarization law take place in the optical and UV domain when the minimum grain size decreases from $200 \mathrm{~nm}$ to $50 \mathrm{~nm}$, with the polarization peak moving from $\sim 1 \mu \mathrm{m}$ to $0.25 \mu \mathrm{m}$, respectively. Analogous results are presented by Voshchinnikov \& Hirashita (2014; their Fig. 2). The application of this line of reasoning to the cases of SN 2006X, 2008fp, and $2014 \mathrm{~J}$, for which $\lambda_{\max }<0.4 \mu \mathrm{m}$, leads to a dust size reduction $>27 \%$. However, the modelling also demonstrates that dust size is not the only ingredient (see below).

It has been suggested for a long time that the shape of the polarization law is related to the extinction law. Serkowski et al. (1975) found that the total-to-selective absorption ratio relates to the position of the polarization peak, following the empirical relation $R_{V}=5.5 \lambda_{\max }$. This was slightly revised by Whittet \& van Breda $\left(1978 ; R_{V}=[5.6 \pm 0.3] \lambda_{\max }\right)$ and Clayton \& Mathis $\left(1988 ; R_{V}=-0.29 \pm 0.74+[6.67 \pm 1.17] \lambda_{\max }\right)$. Inserting $\lambda_{\max } \leq$ $0.4 \mu \mathrm{m}$ into these empirical relations gives the following upper limits on $R_{V}: 2.2,2.2 \pm 0.1$ and $2.3 \pm 0.9$, respectively. All these values are consistent with the spectrophotometric derivations of $R_{V}$ for SN 2006X, 2008fp, and 2014J, although we note that the uncertainties in the Clayton \& Mathis relation are quite large. For SN 1986G, the cited relations give $R_{V}=2.4-2.6$, which fully agrees with the spectrophotometric estimate (Table 1; see also the discussion in Phillips et al. 2013).

The $R_{V}$ vs. $\lambda_{\max }$ relation has been shown to behave differently in different regions of the Galaxy, to the extent that in some cases there is a poor (or even no) correlation between the two quantities (Whittet et al. 2001; Andersson \& Potter 2007). This is interpreted as an indication of a dependence of the grain alignment on its nature, which brings the dust composition into the game. The models show that, at least in the Galaxy, silicates are most likely the only contributors to polarization (see Voshchinnikov 2012, and references therein). Therefore, while the extinction curve (and hence $R_{V}$ ) is determined by dust grains of all species (silicate, carbonaceous, iron-rich, etc.) the polarization law is not. Consequently, under particular conditions, the two can become weakly or even not correlated (Voshchinnikov 2012). For the sake of completeness, we mention that none of the cited works include stars with $\lambda_{\max }<0.39 \mu \mathrm{m}$.

Despite these facts, the four SN we present consistently show values, of both $\lambda_{\max }$ and $R_{V}$, that are very uncommon (or even unprecedented) in our Galaxy. The Galactic sample presented by Valencic et al. (2004) does not include any object with $R_{V} \leq 2$, while Fitzpatrick \& Massa (2007) report only one such star $(\sim 0.3 \%$ of their sample). This fraction increases to $\sim 6 \%$ in the anomalous extinction sample by Mazzei \& Barbaro (2011). The deviating objects are not associated with high reddening $\left(E_{B-V}<0.5\right)$, hence excluding the possibility that the exceptionally low values derived for our $\mathrm{SN}$ are necessarily the product of large extinction. Although $R_{V}$ ratios that significantly deviate from the average are observed in our Galaxy, the distribution is significantly skewed towards large values. In the Fitzpatrick \& Massa sample, the fractions of stars with $R_{V} \geq 3.5$ and $R_{V} \geq 4.0$ are $21.6 \%$ and $9.5 \%$, respectively. Therefore, the exceptional behaviours observed for the Type Ia sample are very hard to interpret in terms of anomalous lines of sight if the host galaxy dust mixtures were similar to that of the MW.

At face value, these findings lead to the striking, antiCopernican conclusion that something is special about the dust of the MW. Another possibility is that the magnetic field of the Galaxy is special. If the field lines are more ordered or the field strength is higher, then the alignment of grains and the resulting polarization would be different. Although not very much is known about extragalactic dust, some information is available. For instance, Finkelman et al. (2010) derived $R_{V}=2.8 \pm 0.4$ for a sample of nine early-type galaxies with dust lanes, and concluded that the extragalactic dust properties are similar to those typical for the Galaxy. This would suggest that there is something special with the lines of sight associated with some Type Ia SN.

The polarization dependencies we report are compatible (at least qualitatively) with dichroic polarization by mixtures with an enhanced amount of small particles with respect to the typical MW distribution. In this respect, to explain the extinction curve of NGC 3034 (M 82), Hutton et al. (2014) suggest that small grains are entrained in the supernovae-driven wind region and would reflect the general properties of the galaxy ISM. However, we emphasize that although the models with reduced 
dust sizes can reproduce the required $\lambda_{\max }$ shifts (Siebenmorgen et al. 2014; Voshchinnikov \& Hirashita 2014), they cannot reproduce the increased slope observed in the blue. This is particularly true for SN 2014J.

The question as to why the galactic environments of these $\mathrm{SN}$ are systematically different from what is seen in similarly reddened stars in the Galaxy remains unanswered. Here we only emphasize that the MW dust properties are deduced from observations of the solar neighbourhood. No line of sight through any external galaxy will geometrically ever be similar to any line of sight from the Earth through the MW.

\subsection{Alternative explanations}

As shown by Wang (2005), Patat et al. (2006), and Goobar (2008), unusually low values of $R_{V}$ can be produced by scattering by circumstellar dust. Although, in principle, this scenario provides a viable explanation, it leaves a number of open issues. Most importantly, the propagation of the light echo through the local dust is expected to produce rapid time variability, both in the resulting $R_{V}$ and in the polarization (in case the dust has an asymmetric geometry). This is particularly true in the early phases, where significant variations are expected on the timescales of a week (Wang 2005; Patat et al. 2006). Second, in order to have a measurable effect on $R_{V}$, this requires conspicuous amounts of dust in the SN vicinity. This poses problems both in terms of dust survival and in the implied presence of significant amounts of gas which, with the possible exception of SN 2006X, were not detected (see Patat et al. 2006 for a detailed discussion on the subject). In the cases of 2006X and 2008fp, in any case, it is difficult to believe that those thick molecular clouds, where the bulk of reddening and polarization are expected to arise (Cox \& Patat 2008, 2014), are directly associated with the SN. A similar argument applies to the numerous components detected along the lines of sight to $1986 \mathrm{G}$ and $2014 \mathrm{~J}$.

The peculiarities of the extinction law along the line of sight to SN 2014J are discussed by Amannulah et al. (2014). They conclude that these are compatible with a power-law extinction with an index close to -2 , as expected from multiple scattering of light (Patat et al. 2006; Goobar 2008). This conclusion is hard to reconcile with the above considerations, as it would require that most of the dust responsible for the observed extinction is confined to distances $\lesssim 10^{16} \mathrm{~cm}$ from the SN. In addition, the large optical depth implied by the measured extinction $\left(\tau_{\text {dust }} \approx 2\right.$ ) would translate into a considerable contribution by multiple scattering, which is an efficient depolarizing mechanism. In these circumstances, the linear polarization is expected to show a steady increase from $0.3 \mu \mathrm{m}$ to $1.0 \mu \mathrm{m}$ (see, for instance, White 1979; Voshchinnikov \& Karjukin 1994; Kartje 1995; Zubko \& Laor 2000), a behaviour that is observed in reflection nebulae (Zellner 1974). This is incompatible with the wavelength dependencies discussed here.

Based on multi-wavelength data including UV spectra, Foley et al. (2014) reached the conclusion that the peculiarities observed in the extinction curve of SN 2014J can be explained by a hybrid model. In this scenario, the reddening, which is found to vary with time, is produced by both circumstellar and interstellar dust, contributing in roughly similar fractions. While the interstellar component has rather normal characteristics $\left(R_{V} \sim 2.6\right)$, the circumstellar dust has LMC-like properties and is described by a power-law extinction with an index very close to -2 , as proposed by Amanullah et al. (2014).

In both scenarios, scattering would play a very relevant role and its imprints should be detectable in the polarization

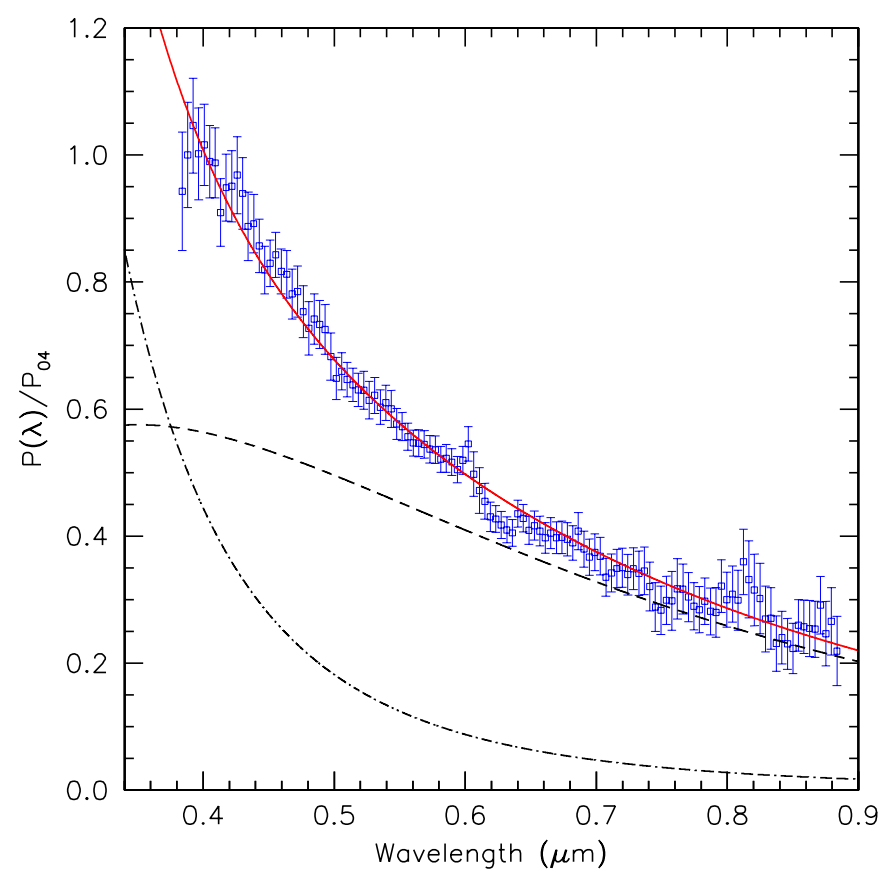

Fig. 6. Illustrative decomposition of the observed polarization in SN 2014J using a Serkowsky law (dashed) and a Rayleigh law (dotteddashed). The solid line fitting the data traces the sum of the two (see text).

signal. In this respect it is worth discussing the cases of the two Galactic stars 19 Tau (Matsumura et al. 2011) and \#46 in IC 63 (Andersson et al. 2013). Both objects are immersed in a reflection nebula and their polarization is most likely affected by a significant scattering component. While 19 Tau shows a slow and steady linear increase in the polarization from red to blue, \#46 in IC 63 displays a very steep rise at short wavelengths. The polarization dependence of \#46 in IC 63 is well reproduced by a $P(\lambda) \propto \lambda^{-4}$ law in the blue range, as expected for both Rayleigh scattering on molecules and Mie scattering in the small grain limit (Andersson et al. 2013). This is coupled to a marked wavelength dependence of the polarization angle, which changes by $\sim 40$ deg from the $B$ to the $U$ passband (Andersson et al. 2013, their Fig. 10). This variation can definitely be excluded in the cases of $1986 \mathrm{G}, 2006 \mathrm{X}$, and $2008 \mathrm{fp}$, while for $2014 \mathrm{~J}$ this is very unlikely (see Fig. 1). This indicates that if a scattering component is present, it behaves differently from known cases in the Galaxy or its polarization angle is, for some reason, parallel to that induced by dichroism.

For illustrative purposes and following Andersson et al. (2013), we applied the decomposition of the observed polarization using both a Serkowsky law and a scattering component to the most extreme case of SN 2014J. For the scattering we adopted the following formulation:

$P(\lambda)=P_{\mathrm{s}}\left(\frac{0.4}{\lambda}\right)^{4}$

where $\lambda$ is expressed in $\mu \mathrm{m}$ and we assumed that the polarization induced by scattering is parallel to that generated by dichroism. The results are shown in Fig. 6. A reasonable fit can be reached using the standard value for $K(1.15), \lambda_{\max }=0.35 \mu \mathrm{m}$ and $P_{\mathrm{s}} / P_{\max }=0.8$. Therefore, although a rather blue $\lambda_{\max }$ is required, a good match can be reached invoking the presence of a scattering component that contributes to about $45 \%$ of the polarization at $0.4 \mu \mathrm{m}$. We emphasize that this is a purely 
phenomenological decomposition. The wavelength dependency will have to be verified with detailed modelling, taking the physical properties of dust into account. These properties include the wavelength dependency of the forwards scattering degree that, in turn, depends on the scattering angle.

Although this finding is intriguing and in line with the scenario proposed by Foley et al. (2014), there is an aspect that needs to be considered in addition to those discussed earlier.

In the case in which the dust is mostly placed at very large distances from the $\mathrm{SN}$, the polarization is acquired purely by transmission (i.e. through dust dichroism) and the polarization angle reflects the properties of the galactic magnetic field via the dust grain alignment. The picture becomes significantly different if a relevant fraction of the dust is confined to the immediate surroundings of the progenitor system. In these circumstances, a non-negligible contribution to polarization would come from photons scattered into the line of sight because scattering is a very efficient polarization mechanism (see Patat 2005, and references therein). In that scenario, the net polarization depends on the geometrical dust distribution (the polarization is perpendicular to the scattering plane). On the contrary, the polarization is null in the case the geometry is spherically symmetric. In all other cases, the resulting net polarization would carry geometrical imprints through the polarization angle, which for circumstellar material has no relation to the magnetic field of the host. The fact that the measured position angles are well aligned with the spiral structure of the hosts places a stringent constraint on the CSM geometry.

Although the presence of a scattering component in our polarization data cannot be ruled out ${ }^{1}$, the scenario proposed by Foley et al. (2014) for SN 2014J needs to be considered in light of the results presented here (lack of time variability in the polarization level and orientation of the electric field). For instance, the Foley et al. (2014) scenario could be reconciled with the polarimetric data if the orientation of the asymmetric CSM dust responsible for the scattering component is, by coincidence, not too different from that of the host magnetic field ${ }^{2}$. The polarization angle measured in SN 2014J is constant to within \pm 5 deg across the whole observed wavelength range (see Fig. 1). This implies that the two orientations indeed need to be very similar. While we cannot rule this out for the specific case of 2014J, it seems unlikely that this is also true for SN 2006X and 2008fp. Based on similar arguments, Kawabata et al. (2014) reached a very similar conclusion. Finally, Brown et al. (2014), on the basis of optical and UV spectrophotometry, argue that, for the specific case of SN 2014J, most of the reddening must be produced by interstellar dust.

Future studies of this kind will need to take into account the important point that dust composition and size distribution are not fixed in time (Hutton et al. 2014). While large grains produced by stars initially dominate the size distribution, this is controlled by ISM processes (Asano et al. 2013a,b, 2014) later on in galaxy history. As a consequence, the extinction curve is flatter in the earliest stages of galaxy evolution. The shape of the extinction curve may be related to the phenomena that lead to dust formation (SN II, AGB stars) and dust destruction ( $\mathrm{SN}$ shocks, metal accretion onto dust grains, etc.). Asano et al. (2013b) find that the dust mass growth in the ISM becomes effective when the metallicity in a galaxy exceeds a critical value.

\footnotetext{
1 In this respect, faint interstellar light echoes were recently resolved by HST (Crotts 2014).

2 The maximum deviation between two linear polarization angles is $90 \mathrm{deg}$.
}

This threshold is larger for a shorter star-formation timescale. Therefore, it is possible that the anomalous behaviour we report is telling us something about the chemical evolutionary state of the galaxy, or areas of the galaxy, in which the explosions occur. We must also take grain growth by accretion and coagulation (Voshchninnikov \& Hirashita 2014) and alignment mechanisms (radiation and paramagnetic) on small dust grains (see for instance Hoang et al. 2014) into account.

\section{Conclusions}

In this article we discussed the wavelength dependence of the insterstellar linear polarization along the lines of sight to four SN Ia and its implications on the dust properties. The conclusions of our study can be summarized as follows:

1. All four $\mathrm{SN}$ included in the sample display an anomalous interstellar polarization law.

2. While SN $1986 \mathrm{G}$ shows a behaviour that is compatible with the most extreme cases known in the Galaxy, SN 2006X, 2008fp, and 2014J display an unprecedented wavelength dependence, with $\lambda_{\max }<0.4 \mu \mathrm{m}$.

3. The total-to-selective absorption ratio $R_{V}$ derived from spectrophotometry is exceptionally low for all four events.

4. In SN 2006X and 2008fp, the bulk of the polarization most likely arises within thick molecular clouds. The anomalous polarimetric behaviour may be associated with the peculiar properties of these clouds.

5. In SN 1986G and 2014J, the polarization is more probably generated within multiple components of similar optical depth along the line of sight. For these events, it is hard to argue in favour of peculiar properties of a single cloud. More plausibly, the observations reflect global characteristics of the hosts.

6. The very small size of the sample does not allow us to establish a relation between the anomalous polarimetric properties and the morphological type of the hosts.

7. Although the objects in the sample are significantly reddened $\left(0.6 \leq E_{B-V} \leq 1.4\right)$, this does not explain the observed behaviour in terms of systematic effects related to high dust optical-depth lines of sight.

8. The dust responsible for the anomalous polarization properties is most probably of interstellar nature. A similar conclusion was reached by Kawabata et al. (2014).

9. Although the presence of circumstellar dust cannot be excluded, the lack of time evolution and the alignment with the local spiral arm pattern pose rather stringent constraints on its geometrical distribution.

10. The extreme case of SN 2014J can be explained, at least phenomenologically, invoking a significant contribution by scattering, provided that an ad hoc distribution for the local dust is adopted.

In general, the observed polarization properties (at least for SNe 1986G, 2006X and 2008fp) can be understood in terms of an enhanced abundance of small grains with respect to the typical MW dust mixture. However, dust size alone cannot explain the observed wavelength dependence. The reasons why all SN in the sample show a behaviour so different from that seen in the Galaxy are unclear. The chemical/physical evolution of the hosts may be playing a relevant role. Future studies will have to proceed on two different fronts: a) increase the sample of well studied Type Ia SN to investigate in a statistically robust way possible correlations between dust properties, galaxy types, and 
SN characteristics; b) obtain high-quality spectropolarimetry of low $R_{V}$ Galactic objects to establish possible connections to what is seen in extragalactic environments.

Acknowledgements. This paper is dedicated to the memory of Prof. Guido Barbaro, who first introduced F.P. to the physics of the interstellar medium. Mandi, Guido. The authors are grateful to Dr G.B. Andersson for illuminating discussions. The German-Spanish Astronomical Center, Calar Alto (Spain) is jointly operated by the Max-Planck-Institut für Astronomie Heidelberg and the Instituto de Astrofisica de Andalucia (CSIC). The Mercator Telescope is operated on the island of La Palma by the Flemish Community, at the Spanish Observatorio del Roque de los Muchachos of the Instituto de Astrofisica de Canarias. The HERMES spectrograph is supported by the Fund for Scientific Research of Flanders (FWO), Belgium, the Research Council of KU Leuven, Belgium, the Fonds National de la Recherche Scientifique (F.R.S.-FNRS), Belgium, the Royal Observatory of Belgium, the Observatoire de Genève, Switzerland, and the Thüringer Landessternwarte Tautenburg, Germany. The authors are grateful to the Calar Alto Observatory and its staff for carrying out the observations in a very efficient way. S.T. acknowledges support by the Trans-regional Collaborative Research Centre TRR33 of the German Research Foundation (DFG). The research of J.C.W. is supported in part by NSF Grants AST-1109801 and No. PHYS-1066293, and the hospitality of the Aspen Center for Physics extended to J.C.W.

\section{References}

Amanullah, R., Goobar, A., Johansson, J., et al. 2014, ApJ, 788, 21 Andersson, B.-G., \& Potter, S. B. 2007, ApJ, 665, 369

Andersson, B.-G., Piirola, V., De Buizer, J., et al. 2013, ApJ, 775, 84

Anderson, C. M., Weitenbeck, A. J., Code, A. D., et al. 1996, AJ, 112, 2276

Appenzeller, I., Fricke, K., Fürtig, W., et al. 1998, The Messenger, 94, 1

Asano, R. S., Takeuchi, T. T., Hirashita, H., \& Nozawa, T., 2013a, MNRAS, 432, 637

Asano, R. S., Takeuchi, T. T., Hirashita, H., \& Inoue, A. K., 2013b, EP\&S, 65, 213

Asano, R. S., Takeuchi, T. T., Hirashita, H., \& Nozawa, T. 2014, MNRAS, 440, 134

Brown, P. J., Smitka, M. T., Wang, L., et al. 2014, ApJ, submitted [arXiv: 1408.2381]

Coyne, G. V., \& Gehrels, T. 1966, AJ, 71, 355

Chotard, N., Gangler, E., Aldering, G., et al. 2011, A\&A, 529, L4

Clayton, G. C., \& Mathis, J. S. 1988, ApJ, 327, 911

Cox, N. L. J., \& Patat, F. 2008, A\&A, 485, 9

Cox, N. L. J., \& Patat, F. 2014, A\&A, 565, A61

Cox, N. L. J, Davis, N., Patat, F., \& Van Winckel, H., 2014, ATEL, 5797

Crotts, A. 2014, ApJ, submitted [arXiv: 1409.8671]

Dolan, J. F., \& Tapia, S. 1986, PASP, 98, 792

D’Odorico, S., di Serego Alighieri, S., Pettini, M., et al. 1989, A\&A, 215, 21

Finkelman, I., Brosch, N., Kniazev, A. Y., et al. 2010, MNRAS, 409, 727

Fitzpatrick, E. L., \& Massa, D. 2007, ApJ, 663, 320

Foley, R. J., Fox, O. D., McCully, C., et al. 2014, MNRAS, 443, 2887

Goobar, A. 2008, ApJ, 686, L103

Goobar, A., Johansson, J., Amanullah, R., et al. 2014, ApJ, 784, L12

Greaves, J. S., Holland, W. S., Jennes, T., \& Hawarden, T. G. 2000, Nature, 404, 732

Hoang, T., Lazarian, A., \& Martin, P. G. 2014, ApJ, 790, 6

Hough, J. .H., Mailey, J. A., Rouse, M. F., \& Whittet, D. C. B. 1987, MNRAS, 227,1

Hutton, S., Ferreras, I., Wu, K., et al. 2014, MNRAS, 440, 150

Jones, T. J. 2000, AJ, 120, 2920
Kartje, J. F. 1995, ApJ, 452, 565

Kawabata, K. S., Akitaya, H., Yamanaka, M., et al. 2014, ApJ, 795, L4

Larson, K. A., Whittet, D. C. B., \& Hough, J. H., 1996 ApJ, 472, 755

Leonard, D. C., \& Filippenko, A. V. 2001, PASP, 113, 920

Leonard, D. C., Filippenko, A. V., Chornock, R., \& Li, W. 2002, ApJ, 124, 2506

Leonard, D. C., Li, W., Filippenko, A. V., Foley, R. J., \& Chornock, R. 2005, ApJ, 632, 450

Mathewson, D. S., \& Ford, V. L. 1970, MNRAS, 74, 139

Maund, J. R., Wheeler, J. C., Patat, F., et al. 2007, MNRAS, 381, 201

Maund, J. R., Wheeler, J. C., Wang, L., et al. 2010, ApJ, 722, 1162

Maund, J. R., Spyromilio, J., Höflich, P. A., et al. 2013, MNRAS, 433, 20

Martin, P. G., Adamson, A. J., Whittet, D. C. B., et al. 1992, ApJ, 392, 691

Martin, P. G., Clayton, G. C., \& Wolff, M. .J. 1999, ApJ, 510, 905

Matsumura, M., Kameura, Y., Kawabata, K. S., et al. 2011, PASP, 63, L43

Mazzei, P., \& Barbaro, G. 2011, A\&A, 527, A34

Meisenheimer, K. 1998, User Guide to the CAFOS 2.2 Astronomische Gesellschaft Abstract Series, 58

Nobili, S., \& Goobar, A. 2008, A\&A, 487, 19

Patat, F. 2005, MNRAS, 357, 1161

Patat, F., \& Romaniello, M., 2006, PASP, 118, 146

Patat, F., \& Taubenberger, S. 2011, A\&A, 529, A57 (PT11)

Patat, F., Benetti, S., Cappellaro, E., \& Turatto, M., 2006, MNRAS, 369, 1949

Patat, F., Baade, D., Höflich, P., et al. 2009, A\&A, 508, 229

Patat, F., Maund, J. R., Benetti, S., et al. 2010, A\&A, 510, A108

Patat, F., Höflich, P., Baade, D., et al. 2012, A\&A, 545, A7

Patat, F., Taubenberger, S., Baade, D., et al. 2014, ATEL, 5830

Phillips, M. M., Simon, J. D., Morrell, N., et al. 2013, ApJ, 779, 38

Scarrot, S. M., Ward-Thompson, D., \& Warren-Smith, R. F. 1987, MNRAS, 224, 299

Serkowski, K. 1974, in Planets, Stars, and Nebulae: Studied with Photopolarimetry, ed. T. Gehrels (University of Arizona Press), IAU Colloq., 23, 135

Serkowski, K., Matheson, D. S., \& Ford, V. L. 1975, ApJ, 196, 261

Siebenmorgen, R., Voshchinnikov, N. V., \& Bagnulo, S. 2014, A\&A, 561, A82

Smith, P. S., Williams, G. G., Smith, N., et al. 2011, ApJ, submitted [arXiv: 1111.6626]

Valencic, L. A., Clayton, G. C., \& Gordon, K. D. 2004, ApJ, 616, 912

Veron, M.-P., \& P. Veron 2006, A\&A, 455, 773

Voshchinnikov, N. V. 2012, J. Quant. Spectr. Rad. Transf., 113, 2334

Voshchinnikov, N. V., \& Hirashita, H. 2014, MNRAS, 445, 301

Voshchinnikov, N. V., \& Karjukin, V. V. 1994, A\&A, 288, 883

Voshchinnikov, N. V., Das, H. K., Yakovlev. I. S., \& Il'in, V. B. 2013, Astron. Lett., 39, 421

Wang, L. 2005, ApJ, 635, L33

Wang, L., \& Wheeler, J. C. 2008, ARAA, 46, 433

Wang, L., Baade, D., Höflich, P., et al. 2003, ApJ, 591, 1110

Weitenbeck, A. J. 2008, Acta Astron., 58, 433

Welty, D. E., Ritchey, A. M., Dahlstrom, J. A., \& York, D. G. 2014, ApJ, 792, 106

Wilking, B. A., Lebofsky, M. J., \& Rieke, G. H. 1982, AJ, 87, 695

White, R. L. 1979, ApJ, 230, 116

Whittet, D. C. B. 2003, Dust in the Galactic Environments, 2nd edn. (Bristol: Institute of Physics Publishing)

Whittet, D. C. B., \& van Breda, I. G. 1978, A\&A, 66, 57

Whittet, D. C. B., Martin, P. G., Hough, J. H., et al. 1992, ApJ, 386, 562

Whittet, D. C. B., Gerakines, P. A., Hough, J. H., \& Shenoy, S. S. 2001, ApJ, 547,872

Zelaya, P., Quinn, J. R., Baade, D., et al. 2013, AJ, 145, 27

Zellner, B. 1974, in Planets, Stars, and Nebulae: Studied with Photopolarimetry, ed. T. Gehrels (University of Arizona Press), IAU Colloq., 23, 867

Zubko, V. G., \& Laor, A. 2000, ApJ, 128, 245 